\title{
Perfil do comportamento de escolares vítimas de bullying e de agressores
}

\author{
Profile of the behavior of schoolchildren victims of bullying and their aggressors \\ Perfil de comportamiento de los escolares víctimas de bullying y de agresores
}

Ismael Schenkel $^{\mathrm{I}}$, Leticia Borfe ${ }^{\mathrm{II}}$, Heloisa Elesbão ${ }^{\mathrm{III}}$, Sandra Mara Mayer ${ }^{\mathrm{IV}}$

\begin{abstract}
Resumo
O estudo objetivou identificar o perfil do comportamento de escolares vítimas de bullying e de agressores, comparando possíveis diferenças entre os sexos. Estudo transversal com 200 escolares, de ambos os sexos, com idades entre 9 a 15 anos. Para a coleta de dados, utilizou-se um questionário com perguntas relacionadas ao bullying. Os dados foram analisados no programa SPSS v. 23.0, considerando um nível de significância de $\mathrm{p}<0.05$ e expressos em frequência absoluta e relativa. Em sua totalidade, observou-se elevado percentual de escolares que já sofreram algum tipo de agressão $(55,5 \%)$, tendo como principal forma a verbal $(25,0 \%)$, e ocorrendo principalmente no recreio escolar $(27,5 \%)$. Um menino foi o mais citado como agressor $(25,5 \%)$. Os achados indicam alta prevalência do bullying escolar tanto para vítimas quanto para agressores e, dessa maneira, destacam a necessidade de ações sociais voltadas à prevenção de atos agressivos e a promoção da convivência escolar de forma saudável e amistosa.
\end{abstract}

Palavras-chave: Agressão; Violência; Bullying; Escolares

\begin{abstract}
The aim of this study was to identify the behavior profile of schoolchildren victims of bullying and aggressors, comparing possible differences between sexes. Cross-sectional study with 200 students from both sexes, age 9 to 15 . For data collection, a questionnaire with inquiries related to bullying was used. The data was analyzed using the SPSS v. 23.0, considering a significance level of $\mathrm{p}<0.05$ and expressed in absolute and relative frequencies. In its entirety, there was a high percentage of schoolchildren who have already suffered some type of aggression $(55,5 \%)$, having verbal as the main one $(25,0 \%)$ and occurring mainly at school recess $(27,5 \%)$. One boy was the most cited as an aggressor $(25,5 \%)$. The findings indicate a high prevalence of school bullying for both victims and aggressors and thus highlights the need for social actions aimed at preventing aggressive acts and promoting school coexistence in a healthy and friendly manner.
\end{abstract}

Keywords: Aggression; Violence; Bullying; Schoolchildren

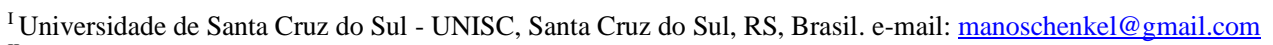

${ }^{\text {II } U n i v e r s i d a d e ~ F e d e r a l ~ d o ~ R i o ~ G r a n d e ~ d o ~ S u l ~-~ U F R G S, ~ P o r t o ~ A l e g r e ~-~ R S, ~ B r a s i l . ~ e-m a i l: ~ b o r f e . l e t i c i a @ g m a i l . c o m ~}$

${ }^{\text {III }}$ Universidade Federal de Santa Maria - UFSM, Santa Maria, RS, Brasil. e-mail: elesbaoheloisa@gmail.com

${ }^{\text {IV }}$ Universidade de Santa Cruz do Sul - UNISC, Santa Cruz do Sul, RS, Brasil. e-mail: smmayer@unisc.br
} 


\section{Resumen}

El estudio tuvo como objetivo identificar el perfil de comportamiento de los escolares víctimas de bullying y agresores, comparando posibles diferencias entre géneros. Estudio transversal con 200 escolares de ambos sexos de 9 a 15 años. Para la recolección de datos se utilizó un cuestionario con preguntas relacionadas con el bullying. Los datos se analizaron mediante el programa SPSS v. 23.0, considerando un nivel de significancia de $\mathrm{p}<0.05$ y expresado en frecuencia absoluta y relativa. En su conjunto, hubo un alto porcentaje de escolares que ya han sufrido algún tipo de agresión $(55,5 \%)$, teniendo la verbal como forma principal $(25,0 \%)$ y ocurriendo principalmente en el recreo escolar (27,5\%). Un niño fue el más citado como agresor (25,5\%). Los hallazgos señalan una alta prevalencia de acoso escolar tanto para víctimas como agresores y así resaltan la necesidad de acciones sociales dirigidas a prevenir actos agresivos y promover la convivencia escolar de manera sana y amigable.

Palabras clave: Agresión; Violencia; Bullying; Escolares

\section{Introdução}

O bullying acontece com frequência em todo o mundo entre os alunos no nível escolar, trazendo resultados negativos. O intuito de tal prática é causar dano às vítimas, tanto psicologicamente, como fisicamente ou emocionalmente. O comportamento violento não pode ser ignorado, pois as vítimas relatam problemas físicos e mentais, ou até problemas de saúde causados pelos abusos sofridos (DZUREC; KENNISON; GILLEN, 2017). Há diferentes tipos de bullying: o físico, o verbal, o relacional e o eletrônico. O bullying físico é relacionado aos atos de empurrar, socar, dar pontapés, chutar, além de furtos, como de lanches e materiais escolares. Esse tipo de violência tende a diminuir de acordo com a idade (BERGER, 2007).

O bullying pode ser definido como uma atitude ou comportamento agressivo acintoso, podendo ser praticado por um ou mais indivíduos, ocorrendo várias vezes, em certo período, contra uma vítima impossibilitada de se defender. Já no ambiente escolar, o bullying pode ser classificado como todo ato de agressão, sendo intencional e repetido, praticado por um ou mais estudantes, contra algum outro indivíduo em situação de desigualdade (SHARIFF, 2011). Além disso, geralmente, os agressores usam as características das vítimas como base para seus atos de violência. Portanto, eles agem contra pessoas que possuem alguma diferença dos demais, como excesso de peso, deficiência física, estatura mais baixa ou mais alta, entre outras características (FANTE, 2005).

Além dos agressores e das vítimas, outros colegas desempenham papéis diferentes durante as práticas de bullying, tanto como ajudantes dos agressores, quanto como defensores das vítimas. Também há os espectadores passivos, aqueles que se retiram de situações em que as violências ocorrem, para que não se tornem as próximas vítimas, o que reforça as atitudes violentas dos agressores (CARAVITA; COLOMBO, 2016). 
O bullying está relacionado aos problemas de saúde pública e pode gerar inúmeros efeitos psicológicos a curto e a longo prazo aos envolvidos. As vítimas das intimidações podem sofrer com estresse, baixa autoestima, ansiedade, depressão, baixo desempenho escolar e, em casos mais graves, até cometem suicídio (COSTA et al., 2015). Em jovens, existe relação significativa entre a exposição ao bullying e o desenvolvimento de depressão e ideação suicida, além de confirmar sua mediação a estes problemas (FUENTES; CARVALLO; POBLETE, 2020).

Para tanto, faz-se necessário identificar e conhecer aqueles que sofrem com as atitudes violentas e aqueles que as praticam no intuito de melhor compreender o fenômeno. Além disso, é indispensável propor ações mais efetivas na solução dos problemas no ambiente escolar. Sendo assim, o presente estudo tem como objetivo identificar o perfil do comportamento de escolares vítimas de bullying e agressores, comparando possíveis diferenças entre os sexos.

\section{Material e métodos}

Este estudo transversal, descritivo e exploratório teve como sujeitos 200 escolares, de ambos os sexos (118 meninos), com idades entre 9 a 15 anos, da rede pública de um município da região central do Rio Grande do Sul. Foram incluídos os escolares matriculados na rede pública, e que assinaram o termo de consentimento livre e esclarecido e termo de assentimento. Utilizou-se como critério de exclusão o não preenchimento por completo do questionário.

Como instrumento de coleta dos dados foi aplicado um questionário de Olweus (1993), adaptado por Mayer (2000), com questões relacionadas ao bullying. Este questionário é composto por blocos, em que o primeiro refere-se à identificação dos participantes, e os blocos restantes, à ocorrência do bullying, forma, lugar das agressões, agressor, inferência do professor e conversa com os pais sobre as agressões.

O presente estudo foi aprovado pelo Comitê de Ética em Pesquisa com Seres Humanos (CEP), sob protocolo CAAE: 86063718.8.0000.5343. Cabe destacar que os participantes com idade maior ou igual a 12 anos assinaram o termo de assentimento (TALE) e os pais ou responsáveis por todos os escolares menores de idade assinaram um termo de consentimento livre e esclarecido (TCLE). Este estudo segue os termos da resolução 466/12 do Conselho Nacional de Saúde (CNS).

Os dados foram digitados e analisados no programa SPSS v. 23.0 (IBM, Armonk, NY, USA), sendo apresentados por meio da estatística descritiva com frequência absoluta e relativa. O teste qui-quadrado foi realizado para verificar o nível de significância, sendo considerando o valor $\mathrm{p}<0.05$. 


\section{Resultados}

Na tabela 1, estão descritas as características dos sujeitos, em sua maior parte do sexo masculino (59\%), com idade de 12 anos $(22,5 \%)$ e pertence ao $7^{\circ}$ ano $(21,5 \%)$.

Tabela 1 - Características gerais dos sujeitos

\begin{tabular}{|c|c|c|c|c|}
\hline & $\begin{array}{c}\text { Masculino }(n=118) \\
n(\%)\end{array}$ & $\begin{array}{c}\text { Feminino }(n=82) \\
n(\%)\end{array}$ & $\begin{array}{c}\text { Total }(\mathbf{n}=200) \\
n(\%) \\
\end{array}$ & $\mathbf{P}$ \\
\hline \multicolumn{5}{|l|}{ Ano } \\
\hline $4^{\circ}$ ano & $17(14,4)$ & $12(14,6)$ & $29(14,5)$ & \multirow{6}{*}{0,191} \\
\hline $5^{\circ}$ ano & $23(19,5)$ & $15(18,3)$ & $38(19,0)$ & \\
\hline $6^{\circ}$ ano & $28(23,7)$ & $12(14,6)$ & $40(20,0)$ & \\
\hline $7^{\circ}$ ano & $26(22,0)$ & $17(20,7)$ & $43(21,5)$ & \\
\hline $8^{\circ}$ ano & $15(12,7)$ & $14(17,1)$ & $29(14,5)$ & \\
\hline $9^{\circ}$ ano & $9(7,6)$ & $12(14,6)$ & $21(10,5)$ & \\
\hline \multicolumn{5}{|c|}{ Idade (anos) } \\
\hline 8 & $1(0,8)$ & $0(0,0)$ & $20(10,0)$ & \multirow{7}{*}{0,589} \\
\hline 9 & $11(9,3)$ & $8(9,7)$ & $19(9,5)$ & \\
\hline 10 & $8(6,7)$ & $11(13,4)$ & $28(14,0)$ & \\
\hline 11 & $17(14,4)$ & $11(13,4)$ & $45(22,5)$ & \\
\hline 12 & $28(23,7)$ & $17(20,7)$ & $34(17,0)$ & \\
\hline 13 & $21(17,8)$ & $13(15,8)$ & $33(16,5)$ & \\
\hline 14 & $19(16,1)$ & $14(17,0)$ & $21(10,5)$ & \\
\hline
\end{tabular}

Fonte: Autoria

Na tabela 2, observa-se que a ocorrência de agressões na sua totalidade é alta (55,5\%), sendo maior entre os meninos $(60,2 \%)$. A agressão verbal é a mais citada $(25,0 \%)$, seguida da violência física (13,5\%). O local onde os incidentes são mais frequentes é no pátio, no momento do recreio (27,5\%), seguidos pelos corredores ou escadas $(13,5 \%)$. Em relação ao agressor, podemos verificar que houve diferença entre os sexos $(\mathrm{p}<0,001)$, sendo que um menino $(25,5 \%)$ ou mais de um menino $(10,0 \%)$ são, na maioria das vezes, os agressores. Quando questionados sobre as interferências dos professores sobre as agressões, 30\% dos alunos relataram que os professores muitas vezes tentam impedir e 30\% citam que, ao sofrer alguma agressão, comunicam os pais. A maioria dos alunos alega que não foram defendidos, por nenhum dos colegas $(27,0 \%)$, ao sofrerem alguma agressão na escola.

Tabela 2 - Ocorrência, forma, momentos e lugar das agressões, quem agrediu, inferência do professor e dos pais sobre as agressões

\begin{tabular}{lcccc}
\hline & $\begin{array}{c}\text { Masculino } \\
\mathbf{n}(\%)\end{array}$ & $\begin{array}{c}\text { Feminino } \\
\mathbf{n}(\%)\end{array}$ & $\begin{array}{c}\text { Total } \\
\mathbf{n}(\%)\end{array}$ & P \\
\hline Você já foi agredido alguma vez na escola? & & & & \\
Sim & $71(60,2)$ & $40(48,8)$ & $111(55,5)$ & 0,147 \\
Não & $47(39,8)$ & $42(51,2)$ & $89(44,5)$ & \\
Forma de agressão & & & & 0,409 \\
Ninguém me agrediu & $49(41,5)$ & $39(47,6)$ & $88(44,0)$ &
\end{tabular}


Bateram, deram socos e chutes

Roubaram coisas

Causaram medo

Agressão verbal

Falaram de mim, contaram segredos meus

Não falaram comigo

Onde te agrediram?

Ninguém me agrediu

No momento do recreio

Nas aulas de Educação Física

Nos corredores ou escadas

$\mathrm{Na}$ sala de aula

\section{Quem te agrediu?}

Ninguém me agrediu

Uma menina

Muitas meninas

Um menino

Muitos meninos

Meninos e meninas

\section{O professor tentou impedir?}

Não sei

Uma

Duas

Muitas vezes

Disseste a teus pais que sofreu agressão?

Ninguém me agrediu

Não contei

Contei

Teus colegas te defendem das agressões?

Ninguém me agrediu

Ninguém me defendeu

Um ou dois colegas me defenderam

Três ou mais colegas me defenderam

$$
\begin{gathered}
21(17,8) \\
5(4,2) \\
8(6,8) \\
27(22,9) \\
6(5,1) \\
2(1,7) \\
\\
49(41,5) \\
37(31,4) \\
7(5,9) \\
16(13,6) \\
9(7,6)
\end{gathered}
$$
$49(41,5)$
$37(31,4)$
$7(5,9)$
$16(13,6)$
$9(7,6)$
$49(41,5)$
$4(3,3)$
$4(3,3)$
$40(33,9)$
$15(12,7)$
$6(5,0)$
$7563,5)$
$7(5,9)$
$5(4,2)$
$31(26,2)$

$51(43,2)$
$28(23,7)$
$39(33,0)$

$43(52,4)$
$18(21,9)$
$21(25,6)$

$41(50,0)$

$10(12,2)$

$6(7,3)$

$11(13,4)$

$5(6,1)$

$9(10,9)$
$27(13,5)$
$7(3,5)$
$12(6,0)$
$50(25,0)$
$12(6,0)$
$4(2,0)$

$89(44,5)$

$55(27,5)$

$10(5,0)$

$\begin{array}{lc}11(13,4) & 27(13,5) \\ 10(12,2) & 19(9,5)\end{array}$

$\begin{array}{lc}11(13,4) & 27(13,5) \\ 10(12,2) & 19(9,5)\end{array}$

0,449

$90(45,0)$

$14(7,0)$

$10(5,0)$

$51(25,5)$

$20(10,0)$

$15(7,5)$

0,001

$\begin{array}{rr}45(54,8) & 120(60,0) \\ 5(6,1) & 12(6,0) \\ 3(3,6) & 8(4,0) \\ 29(35,3) & 60(30,0)\end{array}$

0,576

$0(30,0)$

$94(47,0)$

$46(23,0)$

$60(30,0)$

0,400

\begin{tabular}{lccc}
$42(35,5)$ & $41(50,0)$ & $83(41,5)$ & \\
$33(27,9)$ & $21(25,6)$ & $54(27,0)$ & 0,062 \\
$29(24,5)$ & $9(10,9)$ & $38(19,0)$ & \\
$14(11,8)$ & $11(13,4)$ & $25(12,5)$ & \\
\hline
\end{tabular}

Dados expressos em frequência absoluta e relativa.

Fonte: Autoria

A tabela 3 destaca a ocorrência de agressões realizadas pelos participantes desta pesquisa, em que $20 \%$ dos entrevistados relatam ter agredido alguém algumas vezes. Ao serem questionados sobre a quantidade de colegas de sala de aula que agrediram alguém, a maioria (17,0\%) relatam apenas um colega, no entanto, ao observarmos os resultados separadamente entre meninos e meninas, percebemos que há diferença significativa $(\mathrm{p}<0,011)$, em que, entre os meninos, o agressor caracteriza-se por ser um colega $(22,0 \%)$, já entre meninas, dois ou três colegas $(12,2 \%)$ e quatro ou mais colegas $(12,2 \%) .18,5 \%$ dos escolares citam ter se juntado com outros colegas para agredir alguém que o irritou muito, e $25 \%$ dos alunos disseram que o professor conversou com eles porque agrediram alguém, mas $20 \%$ admitem que os pais não conversaram com eles. 
Tabela 3 - Ocorrência, quem agrediu, inferência do professor e dos pais sobre as agressões

\begin{tabular}{|c|c|c|c|c|}
\hline & $\begin{array}{c}\text { Masculino } \\
\mathbf{n}(\%)\end{array}$ & $\begin{array}{c}\text { Feminino } \\
\mathbf{n}(\%)\end{array}$ & $\begin{array}{l}\text { Total } \\
\text { n( } \%)\end{array}$ & $\mathbf{P}$ \\
\hline \multicolumn{5}{|l|}{ Você já agrediu alguém? } \\
\hline Nenhuma vez/nunca agredi ninguém & $45(38,1)$ & $46(56,1)$ & $91(45,5)$ & \multirow{8}{*}{0,240} \\
\hline Uma vez & $9(7,6)$ & $7(8,5)$ & $16(8,0)$ & \\
\hline Poucas vezes & $21(17,8)$ & $8(9,7)$ & $29(14,5)$ & \\
\hline Algumas vezes & $25(21,1)$ & $15(18,2)$ & $40(20,0)$ & \\
\hline Várias vezes & $6(5,0)$ & $3(3,6)$ & $9(4,5)$ & \\
\hline Muitas vezes & $10(8,4)$ & $3(3,6)$ & $13(6,5)$ & \\
\hline Só para se defender & $1(0,8)$ & $0(0,0)$ & $1(0,5)$ & \\
\hline Não sei & $1(0,8)$ & $0(0,0)$ & $1(0,5)$ & \\
\hline \multicolumn{5}{|c|}{ Quantos alunos da tua sala agrediram outros colegas? } \\
\hline Nenhum & $64(54,2)$ & $54(65,8)$ & $118(59,0)$ & \multirow{4}{*}{0,011} \\
\hline Um colega & $26(22,0)$ & $8(9,7)$ & $34(17,0)$ & \\
\hline Dois ou três colegas & $23(19,4)$ & $10(12,2)$ & $33(16,5)$ & \\
\hline Quatro ou mais colegas & $5(4,2)$ & $10(12,2)$ & $15(7,5)$ & \\
\hline \multicolumn{5}{|c|}{ Te juntaste com outros para agredir alguém? } \\
\hline Não & $83(70,3)$ & $66(80,4)$ & $149(74,5)$ & \multirow{4}{*}{0,157} \\
\hline Só se ele me irrita muito & $23(19,4)$ & $14(17,0)$ & $37(18,5)$ & \\
\hline Não sei & $9(7,6)$ & $1(1,2)$ & $10(5,0)$ & \\
\hline $\operatorname{Sim}$ & $3(2,5)$ & $1(1,2)$ & $4(2,0)$ & \\
\hline \multicolumn{5}{|c|}{ O professor falou contigo por teres agredido alguém? } \\
\hline Não agredi ninguém & $57(48,3)$ & $58(70,3)$ & $115(57,5)$ & \multirow{4}{*}{0,012} \\
\hline Sim, falou & $38(32,2)$ & $12(14,6)$ & $50(25,0)$ & \\
\hline Não falou & $11(9,3)$ & $5(6,1)$ & $16(8,0)$ & \\
\hline Professor não soube & $12(10,1)$ & $7(8,5)$ & $19(9,5)$ & \\
\hline \multicolumn{5}{|c|}{ Teus pais falaram contigo por teres agredido alguém? } \\
\hline Não agredi ninguém. & $62(52,5)$ & $59(71,9)$ & $121(60,5)$ & \multirow{4}{*}{0,032} \\
\hline Uma vez esta semana. & $3(2,5)$ & $1(1,2)$ & $4(2,0)$ & \\
\hline Não falaram. & $26(22,0)$ & $14(17,0)$ & $40(20,0)$ & \\
\hline Sim, falaram. & $27(22,8)$ & $8(9,7)$ & $35(17,5)$ & \\
\hline
\end{tabular}

Dados expressos em frequência absoluta e relativa.

Fonte: Autoria

\section{Discussão}

Neste estudo, 55,5\% dos escolares relatam ter sofrido algum tipo de agressão no ambiente escolar. Diferentemente, estudos realizados no Chile (HIDALGO-RASMUSSEN et al., 2015) e na China (HAN; ZHANG; ZHANG, 2017), identificaram menores percentuais, em que $14,7 \%$ e $26,1 \%$ dos alunos sofreram algum tipo de agressão na escola.

Entre os tipos de agressões sofridas, os dados deste estudo demonstram que a agressão verbal $(25,5 \%)$, seguida da agressão física $(13,5 \%)$, são as mais comuns. Um estudo realizado em Bragança Paulista-SP (MEDEIROS et al., 2015) traz resultados parecidos, no qual 5,7\% dos alunos disseram ter sofrido agressões físicas, já as agressões verbais foram sofridas por 27,5\% dos alunos. Contudo, em um 
estudo realizado em Porto Alegre - RS, os autores identificaram que as agressões verbais têm índices ainda maiores $(61,1 \%)$ (BANDEIRA; HUTZ, 2012). Quanto aos locais onde mais ocorreram os incidentes, observou-se que $27,5 \%$ dos alunos relatou ser no momento do recreio, corroborando com resultados encontrados em estudos realizados na cidade de Vera Cruz-RS (24,8\%) (BRINGMANN, 2016), em Novo Cabrais-RS (23,0\%) (ELESBÃO; BORFE; MAYER, 2016), em Santa Cruz do Sul-RS (12,42\%) (CARVALHO, 2012) e em um município da região central do Rio Grande do Sul (12,4\%) (KEGLER et al., 2020). Nesses estudos, os entrevistados também citaram o recreio como principal momento de ocorrência das agressões.

Ao serem questionados sobre quem os agrediu, de forma geral, a maioria dos participantes desta pesquisa relata ser um menino, sendo que, quando os resultados são comparados entre os sexos, observase que essa indicação permanece $(33,9 \%)$ com diferença significativa no relato do sexo masculino. Estudo realizado em Lavras-MG (ARAUJO; REIS, 2012), apontou que 37\% dos meninos já agrediram algum colega. Alguns estudos associaram o sexo masculino a uma maior tendência de se tornarem autores do bullying (CALBO et al., 2009; RECH et al., 2013). No caso do estudo de Rech et al. (2013), os meninos apresentaram mais do que o dobro de chances de se tornarem agressores, quando comparados às meninas. Os mesmos autores acreditam que a explicação para isso poderia estar no fato de os meninos, muitas vezes, dependendo da idade, encontram-se em uma fase em que a busca pelo reconhecimento, status ou prestígio social frente às meninas, levando-os a cometeram comportamentos violentos e de risco, com o intuito de se destacarem socialmente.

A ligação entre masculinidade e o bullying, conforme Silva, Dascanio e Valle (2016), pode ser elucidado a partir de definições acerca dos modelos culturais, princípios e perspectivas sociais, dando ênfase ao papel do agressor como líder. Esses traços podem estar relacionados à personalidade masculina, tanto em meninos quanto em meninas, levando-os a se tornarem agressivos com seus pares.

Em um estudo realizado em Porto Alegre -RS (BANDEIRA; HUTZ, 2012), os achados indicam que os meninos se identificam mais como agressores do que as meninas, além disso, a maioria das meninas relatou ter sido agredida, principalmente, por meninos. Para os autores, na literatura, muitos estudos apontam o sexo masculino como o principal executor de atos agressivos, no entanto, esses dados não indicam que os meninos são mais agressivos em relação às meninas, mas sim, que são capazes de se envolverem mais e com maior frequência com o bullying, em relação às meninas. Há tempos, Lopes Neto (2005) já levantava uma discussão acerca desse assunto, em que debatia a maior ocorrência de envolvimento dos meninos com o bullying em relação às meninas, contudo sem denotar que os mesmos tenham comportamentos mais agressivos, mas sim, que possuem maior probabilidade de assumir esse tipo 
de comportamento. Soma-se a isso o fato de as meninas adotarem formas de bullying mais difíceis de serem percebidas, como o bullying indireto (LOPES NETO; SAAVEDRA, 2004).

Em estudo de Costa et al. (2015), na cidade de Belo Horizonte-MG, 54,8\% dos alunos relatam aos pais sobre as agressões sofridas na escola, diferentemente do presente estudo, em que somente 30,0\% comunicou o fato em casa. A respeito das atitudes dos professores ao tentarem impedir as agressões, 30,0\% dos alunos relataram que os professores sempre tentam impedir as agressões, sendo que tais índices poderiam ser maiores, comprovado pelo estudo realizado no Distrito Federal por Santos (2010), em que $24,8 \%$ dos alunos relataram que os professores sequer têm conhecimento sobre os ocorridos, sendo este um fator relacionado ao índice do presente estudo.

Segundo estudo de Crema (2014), realizado em Rio Pardo-RS, dos alunos questionados, 48,57\% tentam ajudar como podem algum colega que sofre alguma agressão. Diferentemente do presente estudo, em que 27,0\% afirmaram não receber ajuda de outros colegas. Torna-se preocupante identificar que não há muito auxílio em situações de bullying que ocorrem no ambiente escolar. E, nesse sentido, estudo realizado com objetivo de analisar o discurso espontâneo de adolescentes sobre o bullying na escola, observou certa indiferença entre os mesmos, já que relataram intimidações sofridas em sala de aula consideradas apenas piadas, e que, se alguém está sendo intimidado, é porque isso possivelmente tem mais relação com a vítima do que com o agressor (POSTIGO et al., 2019).

Ao serem questionados sobre a prática do bullying, 20,0\% dos participantes deste estudo, com maior prevalência do sexo masculino, relataram já ter agredido alguém algumas vezes. No entanto, nota-se a dificuldade que os escolares, principalmente os adolescentes, têm em assumir o envolvimento físico no comportamento do bullying, seja por um ato de violência ou por causa do constrangimento em relatar o episódio físico. Além disso, muitos dos adolescentes costumam interpretar o episódio como brincadeira, que então, não é percebido como agressão (REISEN; VIANA; SANTOS-NETO, 2019).

O presente estudo identificou diferenças significativas nas respostas dos alunos, na comparação entre o sexo, em questões sobre um colega de sala ser o agressor, na inferência do professor quando as agressões acontecem e em situações em que os alunos conversam com os pais sobre a situação. Nesses quesitos, a maioria dos meninos identificou apenas um colega como agressor da sala de aula (22,0\%), que o professor falou com eles por terem agredido alguém $(32,2 \%)$ e que os pais falaram com eles pela ocorrência da agressão (22,8\%). A este respeito, faz-se importante a participação dos pais nas pesquisas sobre o fenômeno bullying, e em ações para a prevenção destas agressões, visto que são eles que têm o contato direto com seus filhos fora da escola, podendo auxiliar na compreensão dos fatores motivadores da prática do bullying (BORSA; PETRUCCI; KOLLE, 2015). 
Estudo com o objetivo de desenvolver uma estratégia participativa para prevenir o bullying escolar entre adolescentes propôs um modelo de intervenção que oportunizou a participação ativa dos adolescentes, contribuindo no desenvolvimento de competências geradoras de comportamento pró-sociais, relações empáticas e assertivas, capazes de enfrentar o bullying e transformar o ambiente escolar (BRANDÃO NETO et al., 2020). Ademais, tem-se a escola como promotora do desenvolvimento humano, a qual deve ser um ambiente acolhedor, que respeite os vitimizados e encoraje a cultura da paz e da não-violência. Para que isso ocorra, é preciso que a escola aborde a sensibilização e a conscientização das ações dos educandos, possibilitando um bom clima moral e contribuindo uma qualidade das relações interpessoais neste ambiente. Existe a necessidade de que a escola como um todo reflita sobre as condutas e a forma como as interações sociais ocorrem em seu interior, promovendo ações preventivas e intervenções efetivas para a cooperação mútua entre os que afrequentam (MONTEIRO; ASINELLI-LUZ, 2020).

Ressalta-se a importância da presente pesquisa ao propor um melhor entendimento acerca do fenômeno bullying no ambiente escolar, podendo contribuir também com os programas e políticas públicas existentes acerca da temática no contexto e na comunidade. Contudo, como limitação do estudo, destacase a não identificação do comportamento dos sujeitos e de seus familiares em ambiente extraescolar, bem como a eficiência dos programas e ações já em desenvolvimento no município no combate às práticas violentas, destacando que estas lacunas limitantes desde estudo devem ser objeto a ser explorado em novas investigações.

\section{Conclusão}

O presente estudo identificou alta prevalência de vítimas de bullying e de agressores, sinalizando que ações devem ser implementadas para minimizar as consequências destes atos. A agressão verbal é a mais relatada, ocorrendo, no momento do recreio escolar e por um menino. Em muitos dos casos, existe a inferência do professor e da família; contudo, observa-se que não há muito auxílio em situações de bullying por parte dos colegas das vítimas. Sugere-se, a partir do presente estudo, que esses assuntos sejam mais abordados e discutidos, tanto na rede escolar quanto em casa, enfatizando as consequências que o bullying pode gerar.

\section{Referências}

ARAUJO, R. S.; REIS, F. P. G. Avaliação diagnóstica sobre a ocorrência de bullying em duas escolas estaduais de Lavras, MG: perspectivas para o planejamento em Educação Física escolar. Lecturas: Educación Física y Deportes, v. 17, n. 167, s/p, 2012. 
BANDEIRA, C. M.; HUTZ, C. S. Bullying: prevalências, implicações e diferenças entre os gêneros. Revista Semestral da Associação Brasileira de Psicologia Escolar e Educacional, v.16, n.1, p. 35-44, 2012.

BERGER, Kathleen S. Update on bullying at school: Science forgoten? Developmental Review, v. 27, n.90, p. 90-126, 2007.

BORSA, J. C.; PETRUCCI, G. W.; KOLLE, S. H. A participação dos pais nas pesquisas sobre o bullying escolar. Revista Quadrimestral da Associação Brasileira de Psicologia Escolar e Educacional, São Paulo, v. 19, n. 1, 41-48, 2015.

BRANDÃO NETO, W. et al. Formation of protagonist adolescents to prevent bullying in school contexts. Revista Brasileira de Enfermagem, v. 73, Supl. 1, p.1-8, 2020.

BRINGMANN, Daniela. R. O bullying no ambiente escolar: um estudo comparativo entre uma escola estadual e outra particular de Vera Cruz - RS. 2016. 41f. Monografia (Graduação do Curso de Educação Física), Universidade de Santa Cruz do Sul, Santa Cruz do Sul-RS, 2016.

CALBO, A. S. et al. Bullying na escola: comportamento agressivo, vitimização e conduta pró-social entre pares. Contextos Clínicos, vol. 2, n. 2, jul./dez. 2009.

CARAVITA, S. C. S.; COLOMBO, B. Bullying behavior, youth's disease and intervention: which suggestions from the data for research on bullying in the Brazilian context? Jornal de Pediatria, v. 92, n.1, p. 4-6, 2016.

CARVALHO, Thiago C. A violência escolar e o bullying na realidade de estudantes do $6^{\circ}$ ano à $8^{\text {a }}$ série da Escola José Mânica. 2012. 29f. Monografia (Graduação do Curso de Educação Física) Universidade de Santa Cruz do Sul, Santa Cruz do Sul, 2012.

COSTA, M. R. et al. Bullying among adolescents in a Brazilian urban center - "Health in Beagá" Study. Revista de Saúde Pública, v.49, n.1, p. 1-10, 2015.

CREMA, Eduardo R. Bullying Escolar: um estudo com escolares de $5^{\circ}$ ao $9^{\circ}$ ano da Escola de Ensino Fundamental Barão do Triunfo do município de Rio Pardo - RS. 2014. Monografia (Graduação do Curso de Educação Física), Universidade de Santa Cruz do Sul, Santa Cruz do Sul-RS, 2014.

DZUREC, L. C.; KENNISON, M.; GILLEN, P. The incongruity of workplace bullying victimization and inclusive excellence. Nursing Outlook, v.65, n.5, p.588-596, 2017.

ELESBÃO, H.; BORFE, L.; MAYER, S. M. Bullying no contexto escolar: manifestação de atos agressivos na escola. Uma pesquisa realizada no município de Novo Cabrais - RS. Lecturas: Educación Física y Deportes, v. 20, n. 214, s/p, 2016.

FANTE, Cleo. Fenômeno bullying: como prevenir a violência nas escolas e educar para a paz. Campinas: Versus, 2005.

FUENTES, E. A.; CARVALLO, P. R.; POBLETE, S. R. Acoso escolar (bullying) como factor de riesgo de depresión y suicídio. Revista Chilena de Pediatría, v. 91, n. 3, s/p, 2020.

HAN, Z.; ZHANG, G.; ZHANG, H. School Bullying in Urban China: Prevalence and Correlation with School Climate. International Journal Of Environmental Research And Public Health, v.14, n.10, p.1116-1117, 2017. 
HIDALGO-RASMUSSEN, C. et al. Bullying y calidad de vida relacionada con la salud en adolescentes escolares chilenos. Revista Médica de Chile, v.143, n.6, p. 716-723, 2015.

KEGLER, E. D. et al. Perfil do bullying escolar: Uma comparação entre escolas da rede estadual e municipal de um município da região central do Rio Grande do Sul. Brazilian Journal of Health Review, v.3, n.3, p. 6396-6406, 2020.

LOPES NETO, Aramis A. Bullying comportamento agressivo entre estudantes. Jornal de Pediatria, v. 81, (supl. 5), p. 164-172, 2005.

LOPES NETO, A. A.; SAAVEDRA, L.H. Diga não para o bullying. Rio de Janeiro: ABRAPIA, 2004.

MAYER, Sandra M. Comportamento Agressivo em Escolares de $1^{\circ}$ a $^{\circ}$ série do Ensino Fundamental de Santa Cruz do Sul: uma abordagem através da Teoria dos Sistemas Ecológicos. 2000. 114f. Dissertação (Mestrado em Desenvolvimento Regional - Área Sócio Cultural) Universidade de Santa Cruz do Sul, Santa Cruz do Sul, 2000.

MEDEIROS, E. D. et al. Escala de Comportamentos de Bullying (ECB): Elaboração e Evidências Psicométricas. Psico-USF, v. 20, n. 3, p. 385-397, 2015.

MONTEIRO, M. P. G.; ASINELLI-LUZ, A. Diálogos sobre o bullying escolar e o desenvolvimento humano. Educação por escrito, v.11, n.1, p. 1-13, 2020.

OLWEUS Dan. Bullying at school. Oxford e Cambridge: Blackwell, 1993.

POSTIGO, S. et al. What Do Adolescents Say about Bullying? Anales de Psicología, v. 35, n. 2, p. 251$258,2019$.

RECH, R. R. et al. Prevalence and characteristics of victims and perpetrators of bullying. Jornal de Pediatria, v. 89, n. 2, p. 165-170, 2013.

REISEN, A.; VIANA, M. C.; SANTOS-NETO, E. T. Bullying among adolescents: are the victims also perpetrators? Brazilian Journal of Psychiatry, v. 41, n. 6, p. 518-529, 2019.

SANTOS, Marcos P. O. Fenômeno bullying na Educação Física Escolar: um estudo de caso no Distrito Federal. Lecturas: Educación Física y Deportes, v. 143, n.15, s/p. 2010.

SHARIFF, Shariff. Ciberbullying: questões e solução para a escola, a sala de aula e a família. Porto Alegre: Artmed, 2011.

SILVA, F.; DASCANIO, D.; VALLE, T. G. M. O fenômeno bullying: diferenças entre meninos e meninas. Reflexão e Ação, v. 24, n. 1, p. 26-46, 2016.

\section{Como citar este artigo}

SCHENKEL, I.; et al. Perfil do comportamento de escolares vítimas de bullying e de agressores. Revista Kinesis, Santa Maria, v. 39, p.01-11, 2021.

* O presente trabalho não contou com apoio financeiro de nenhuma natureza para sua realização. 